\title{
A preliminary study on the performance of indoor active noise barriers based on 2D simulations
}

\author{
Xiaofan Huang, Haishan Zou*, Xiaojun Qiu \\ Key Laboratory of Modern Acoustics and Institute of Acoustics, Nanjing University, Nanjing 210093, China
}

\section{A R T I C L E I N F O}

\section{Article history:}

Received 1 April 2015

Received in revised form

28 June 2015

Accepted 30 June 2015

Available online xxx

\section{Keywords:}

Enclosure

Active noise barrier

Reflecting boundary

\begin{abstract}
A B S T R A C T
Single screen barriers are widely used in open-plan offices as a means of improving acoustical privacy. However, the insertion loss of an indoor barrier is limited in low frequency range. Active control systems have been shown to be able to enhance the insertion loss of outdoors noise barrier in low frequency range, so its feasibility for improving the performance of indoor barriers is investigated in this paper. The simulation results show that about $10 \mathrm{~dB}$ extra insertion loss below $500 \mathrm{~Hz}$ can be obtained with the active control system when the squared sound pressure inside the entire observation area is minimized. The performance of the active noise barrier deteriorated significantly due to the reflecting ceiling, and the performance is hardly improved by increasing the ceiling height or the ceiling absorption coefficient. Finally, the effects of the sensing strategy on the control performance and the impacts of the active control system on the source side are discussed.
\end{abstract}

(c) 2015 Elsevier Ltd. All rights reserved.

\section{Introduction}

In order to improve the visual and acoustical privacy in openplan offices, a general practice is to use free-standing screens to separate individual workplaces [1]. Although acoustic properties of passive and active noise barriers in free field have been widely studied, their performance in enclosed spaces have not been fully investigated [2-5]. This paper will investigate the performance of indoor active noise barriers.

In enclosed spaces, sound energy reaches the receiver on the other side of the screen via diffraction at the screen edge and reflections on the boundaries which include ceiling and walls. Acoustic modelling of indoor barriers can be classified into three categories: the methods based on the diffuse-field theory [6,7], the volume or surface integral methods [8-10] and the geometrical methods [11-14].

The first category was initially proposed by Moreland and Musa in 1973 [6]. Taking diffraction into account in this method is genuinely difficult because the sound field is not uniform when moving away from the screen. This difficulty was solved in part by Lau and Tang in 2009 by taking distance effect into account.

\footnotetext{
* Corresponding author. Tel.: +86 258359 4505; fax: +86 2583592919

E-mail address: hszou@nju.edu.cn (H. Zou).
}

However, the solution based on the modified diffuse-field theory is still limited to simple cases [7].

The second category is based on the solution of the Helmholtz equation or its integral form. Kopuz and Lalor proved its accuracy by comparing the results between simulation and experiments in 1995. But this method generally needs long computation time for complicate environment or at high frequencies due to the sophisticated meshes [8]. The radiosity method (radiative transfer of acoustical energy) was initially a surface integral method applied to the acoustic energy instead of the pressure field. Le Bot and Bocquillet applied it successfully to a simple enclosure and Reboul et al. extended it to multiple diffractions [9,10]. However, these middlehigh frequency approaches ignore the spatial interferences of the sound field.

The third category consists of identifying acoustic paths between source and receiver and determining the energy loss caused in the propagation [11]. Wang and Bradley proposed a method based on coherent summing of the image sources for the direct, the reflected and the diffracted fields [12], which was used to calculate intelligibility indicators for acoustic design of open-plan offices [13]. This method is complicated with the presence of more than one screen in the room and the enormous number of image sources. Chevret and Chatillon described a model for predicting diffraction in complex spaces by using the ray-tracing method in 2012, which belongs to the family of incoherent methods. A phaseincluded expression of the existing model was developed recently 
in 2014, but this method has limitations at low frequencies [11,14].

Even with so much effort, these existing methods still cannot solve the acoustics problem of indoor barriers completely. The methods based on the diffuse-field theory are limited by the assumption of uniform distribution of sound energy density and sound absorption, which is seldom fulfilled in reality, particularly in the presence of a barrier in an open-plan office. The volume or surface integral methods need long computation time for complex environment or at high frequencies, while the geometrical methods are usually most effective in rooms with regular highly reflective surfaces at high frequencies.

On the other side, existing analytical and experimental studies have revealed that active control can be used to improve the performance of outdoor noise barriers in low frequency range, where the effects of the ground reflection, the geometry shape of the secondary source, the cost function and the error sensor strategies have been investigated $[2-5,15,16]$. There is also a lot of research on active control of enclosed sound field [17-20]. For example, local active control can be applied inside automobiles and aircrafts to cancel the noise near a listener's ears [21].

An indoor active noise barrier is an active noise barrier in an enclosure. Little research has been undertaken in this direction. This paper will investigate its performance based on 2D simulations, especially the effects from the reflection of the ceiling and walls, the height of the ceiling as well as the sensing strategies in a typical room. The preliminary research should be useful for exploring new approaches to improve speech privacy in open-plan offices with active noise barriers in the future.

\section{Simulation model}

This research uses 2D numerical simulations with the commercial software package COMSOL 4.4 [22], because a complete analytical solution is still not available. The reliability of the model in the COMSOL was verified by comparing the simulation results with the Hadden-Pierce solution based on a semi-free barrier model of $1.5 \mathrm{~m}$ high and $0.01 \mathrm{~m}$ thick [23]. The reason that 3D simulations are not adopted is because the computation time and the required memory at high frequency are too high and the purpose of this research is for understanding the fundamental performance of an indoor active noise barrier instead of a practical design. Fig. 1 shows the $6 \mathrm{~m}$ long by $3 \mathrm{~m}$ wide rectangular space under investigation, where the coordinate origin is chosen at the left bottom corner of the enclosure. For simplicity, the current model does not include the complete workstations but only a single absorptive separating barrier.

A $1.5 \mathrm{~m}$ high and $0.01 \mathrm{~m}$ thick single barrier located at $x=2.0 \mathrm{~m}$ separates the enclosure into two rectangular spaces. The sound



Fig. 1. Geometry of an indoor active noise barrier in an enclosure.
Table 1

Layer parameters of the boundaries of the enclosure model.

\begin{tabular}{lllll}
\hline & Ceiling & Ground & Walls & Barrier \\
\hline Flow resistivity $(\sigma)(\mathrm{cgs})$ & 600 & 800 & 2000 & 1500 \\
Porosity $(\Omega)$ & 0.6 & 0.2 & 0.1 & 0.2 \\
Tortuosity $(T)$ & 1 & 1.4 & 1.1 & 1.2 \\
Pore shape factor $\left(S_{p}\right)$ & 0.25 & 0.25 & 0.1 & 0.2 \\
Thickness $(d)(\mathrm{m})$ & 0.1 & 0.05 & 0.02 & 0.005 \\
\hline
\end{tabular}

transmitted through the screen is negligible by assuming the transmission loss of the barrier to be larger than $20 \mathrm{~dB}$. A primary source with a source strength of $0.01 \mathrm{~m}^{2} / \mathrm{s}$ is placed at $(1.3 \mathrm{~m}, 1.0 \mathrm{~m})$ as a sitting position of a person, and a secondary source used to cancel the noise is arranged on the top of the screen with a distance of $0.01 \mathrm{~m}$. Taking the movement of the receiver into account, a square with a length of $0.4 \mathrm{~m}$ located at $(2.7 \mathrm{~m}, 1.0 \mathrm{~m})$ is selected as the observation area (surrounded by the right dashed line square in Fig. 1).

In order to investigate the performance limits of the indoor passive and active noise barrier, the cost function for determining the optimal secondary source strength is to minimize the sum of the squared sound pressure at the entire observation area. After obtaining the optimal secondary source strength, the sound field distribution after active control is calculated. For simplicity, 4 boundaries are all assumed to be rigidly backed layers of homogeneous porous material. Table 1 shows the flow resistivity $(\sigma)$, porosity $(\Omega)$, tortuosity $(T)$, pore shape factor $\left(S_{p}\right)$ and thickness $(d)$ that are used to evaluate the surface admittance [24]. Fig. 2 shows the calculated corresponding normal incident absorption coefficients of these four boundaries, where the legend "Wall" means the front and back walls in this 2D room.

The performance of the indoor passive and active noise barrier is evaluated in $1 / 3$ octave bands with the average sound pressure level (SPL) of the observation area, which can be calculated by

$\mathrm{SPL}=10 \log _{10}\left[\left(\sum_{i=1}^{N} \frac{1}{M} \sum_{j=1}^{M}\left|p_{j}(i)\right|^{2}\right) / p_{\text {ref }}^{2}\right]$

where $N=10$ is the number of sampling frequencies calculated in a $1 / 3$ octave band, $\left|p_{j}(i)\right|^{2}$ is the squared absolute sound pressure at the $j$ th equally spaced observation point (the total number of points is $M=441$ ) at the $i$ th equally spaced sampling frequency, and $p_{\text {ref }}=$ $2 \times 10^{-5} \mathrm{~Pa}$ is the reference sound pressure.



Fig. 2. Spectra of normal incident absorption coefficient on four boundaries of the enclosure in numerical simulations. 


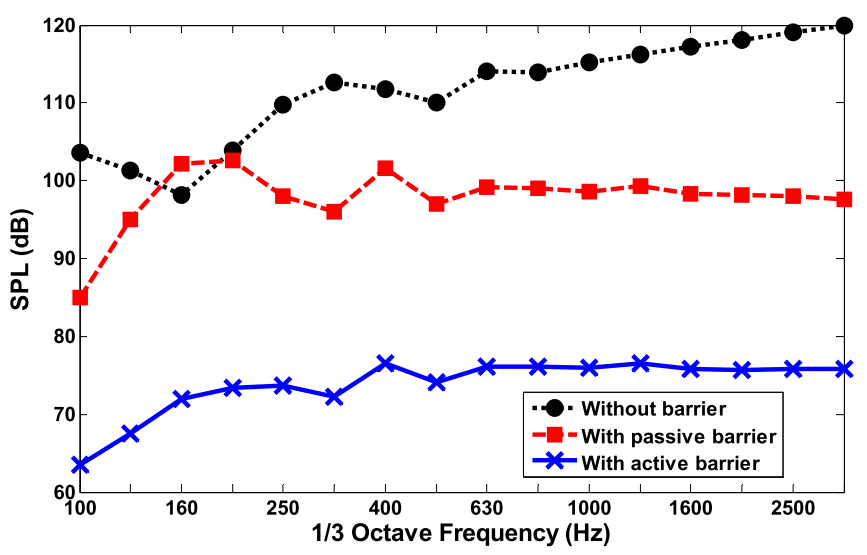

(a)

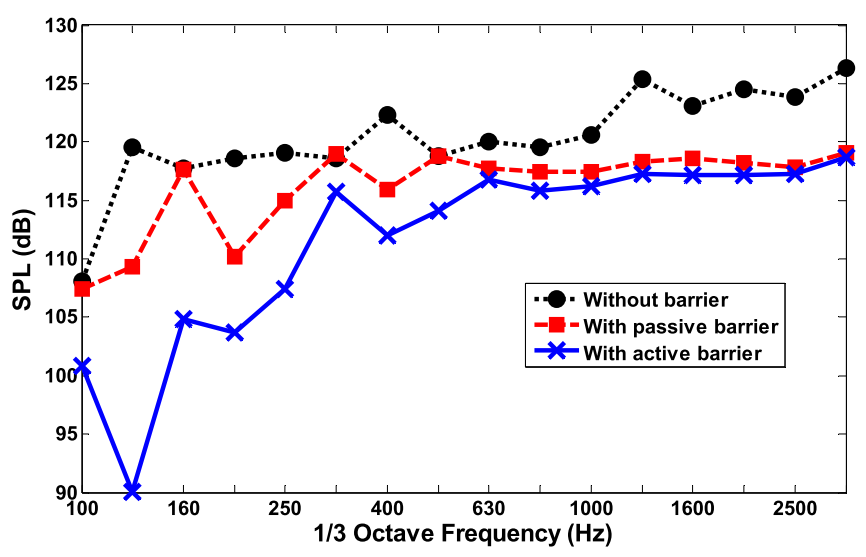

(b)

Fig. 3. Performance of the passive and active noise barrier: (a) sound pressure level in semi-free field; (b) sound pressure level in an enclosure.

\section{Simulation and discussions}

3.1. The performance of the indoor passive and active noise barrier

The performances of the passive and active noise barrier in semi-free field and in the enclosed space are shown in Fig. 3. With the barrier in semi-free space, the SPL in the observation area has been significant reduced. As shown in Fig. 3(a), the passive barrier obtains more than $10 \mathrm{~dB}$ insertion loss above $250 \mathrm{~Hz}$, but little noise

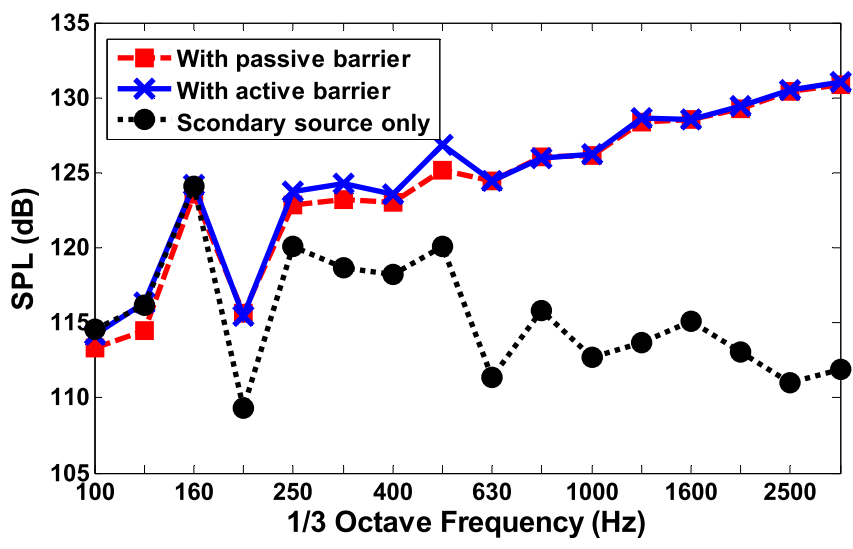

Fig. 4. The sound pressure level on the area of the source side. reduction around $160 \mathrm{~Hz}$ and $200 \mathrm{~Hz}$. This is due to the destructive interference of different diffraction paths around this frequency range. About more than $20 \mathrm{~dB}$ extra insertion loss is obtained in the entire frequency range with the active control system. The overall SPLs without the barrier, with the passive barrier, and with the active barrier (means the passive barrier and the active control system function together) are $126.8 \mathrm{~dB}, 111.0 \mathrm{~dB}$, and $86.7 \mathrm{~dB}$, respectively.

When the barrier is located in the enclosed space, as shown in

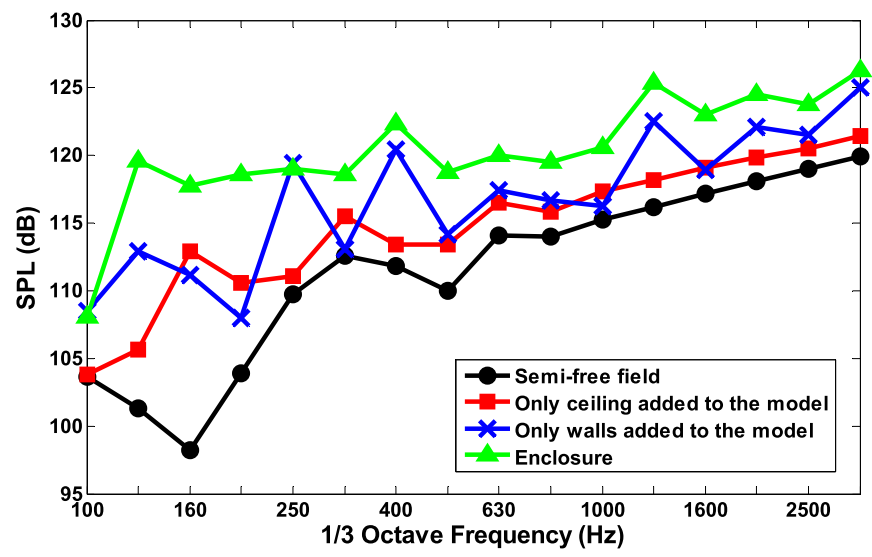

(a)

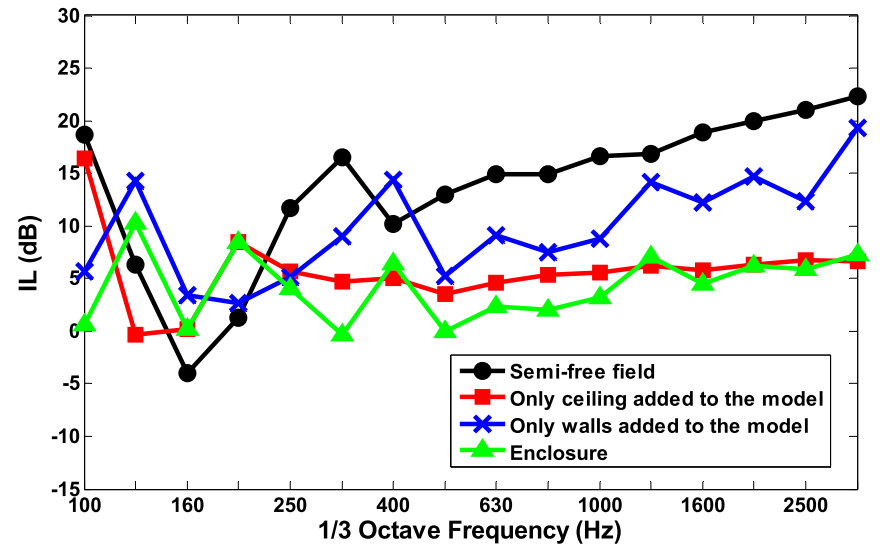

(b)

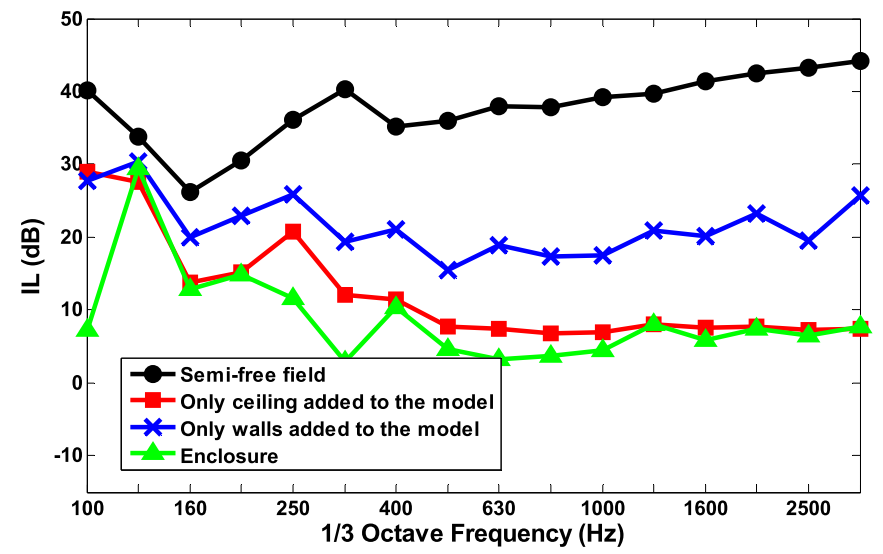

(c)

Fig. 5. Performance of the passive and active noise barrier at different reflecting conditions: (a) sound pressure level without barrier; (b) insertion loss of the passive barrier; (c) insertion loss of the active barrier. 


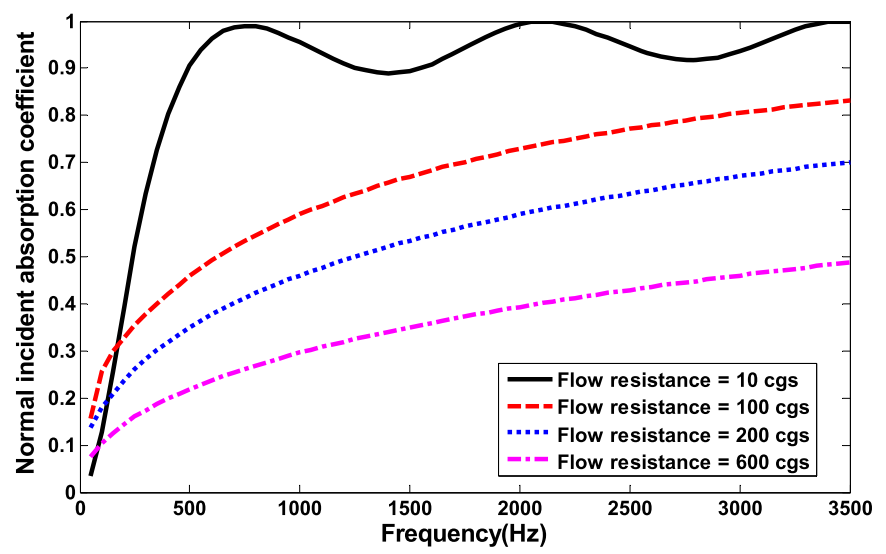

Fig. 6. Spectra of normal incident absorption coefficient of the ceiling at 4 flow resistance in numerical simulations.

Fig. 3(b), the noise reduction performance of the barrier deteriorates significantly. Because of reflections from the boundaries of the enclosure, the SPL of the primary field in the enclosure becomes larger, and the performance of the indoor passive and active noise barrier decreases. The indoor passive barrier insertion loss at most frequency below $1000 \mathrm{~Hz}$ was less than $5 \mathrm{~dB}$. The overall SPLs without the barrier, with the passive barrier, and with the active barrier are $133.9 \mathrm{~dB}, 129.2 \mathrm{~dB}$, and $127.0 \mathrm{~dB}$, respectively. The active system dramatically improves the passive barrier performance in the low frequency range, about 5-20 dB extra insertion loss is obtained below $630 \mathrm{~Hz}$, but it has little improvement in high frequency range.

Because the primary source is at a position of a person, the impact of the secondary source of the active control system on the source side is important. A square area (the left dotted line square in Fig. 1) with a side length of $0.4 \mathrm{~m}$ located at the primary source side is chosen as the observation area, where the average SPL evaluated in $1 / 3$ octave bands is used to assess the impact of the indoor active noise barrier on the source side. Fig. 4 shows the SPL difference on the source side with the active system on and off, where it can be observed that the difference is less than $1 \mathrm{~dB}$, so the impact of the indoor active noise barrier on the area of the source side is small and should not be noticed generally. The reason that the effect of the indoor active noise barrier is generally small on the source side is because the secondary source only generates the power to cancel the diffracted sound, which is usually just a fraction of the original sound, as shown by the black dotted line in Fig. 4.

\subsection{The influence of the reflecting boundaries}

The deterioration of the performance due to the reflecting boundaries is examined by comparing the SPL and insertion loss (IL) with different reflection conditions. In the simulations, the ground and the barrier exist in all cases, and this means that all simulation models in this subsection are composed of the semi-free field model with partially reflective surfaces with different configurations.

As shown in Fig. 5(a), with increasing reflections from the boundaries, the sound pressure level at the observation area increases, but the noise reduction performance of the passive and active barriers deteriorates significantly, as shown in Fig. 5(b) and (c). The ceiling reflection has a dominating influence on the barrier performance, which resulting in a larger than $10 \mathrm{~dB}$ deterioration not only on the passive barrier but also on the active barrier. The reflective boundaries have a greater impact on the active barrier than on the passive barrier, but the active system still has a considerable better performance in low frequency range.

\subsection{The effect of the ceiling reflection}

To understand of the influence of the ceiling reflection better, simulations are carried out in a flat room with a height of $3.0 \mathrm{~m}$. Except the ceiling, all other parameters have the same values as that in Section 2. The flow resistance of the ceiling is changed and

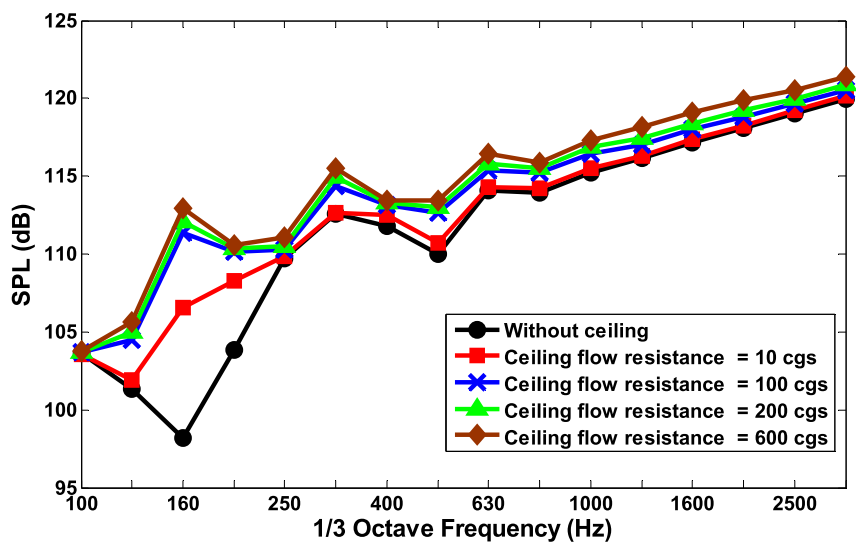

(a)

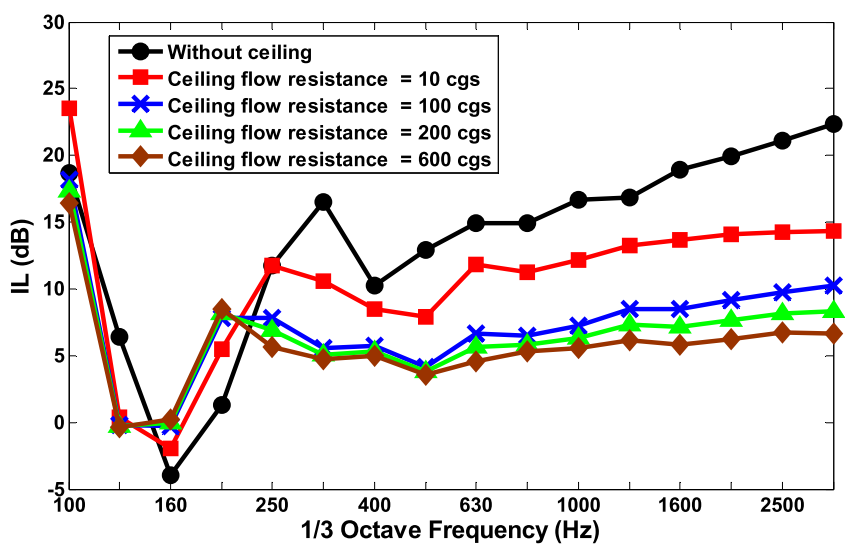

(b)

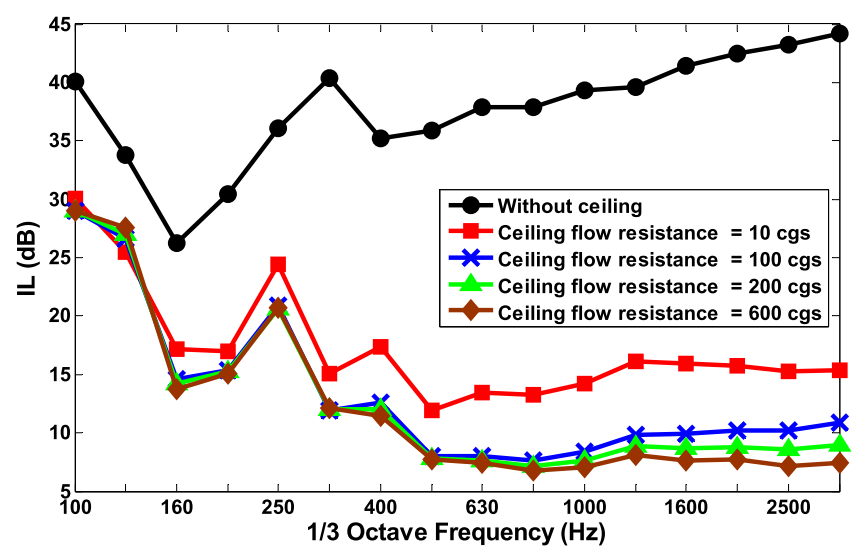

(c)

Fig. 7. Performance of the passive and active noise barrier at different ceiling absorption conditions: (a) sound pressure level without barrier; (b) insertion loss of the passive barrier; (c) insertion loss of the active barrier. 
the calculated normal incident absorption coefficients are shown in Fig. 6.

Fig. 7 shows the SPLs and ILs in these 4 different ceiling cases, where it is found that the smaller the ceiling absorption coefficient is, the greater the deterioration will be. The existence of the ceiling has a significant impact on the active barrier than on the passive barrier. With the ceiling, the performance of the active system deteriorates more than $20 \mathrm{~dB}$. Increasing the ceiling absorption

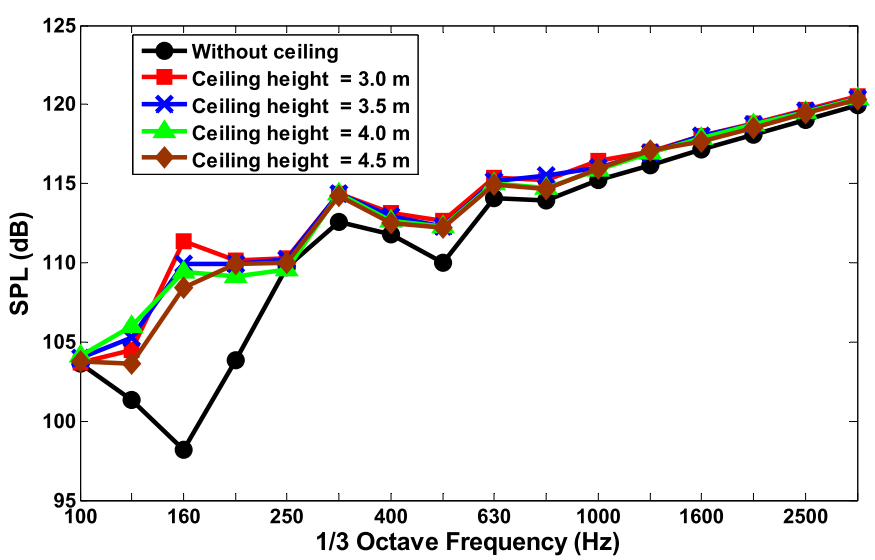

(a)

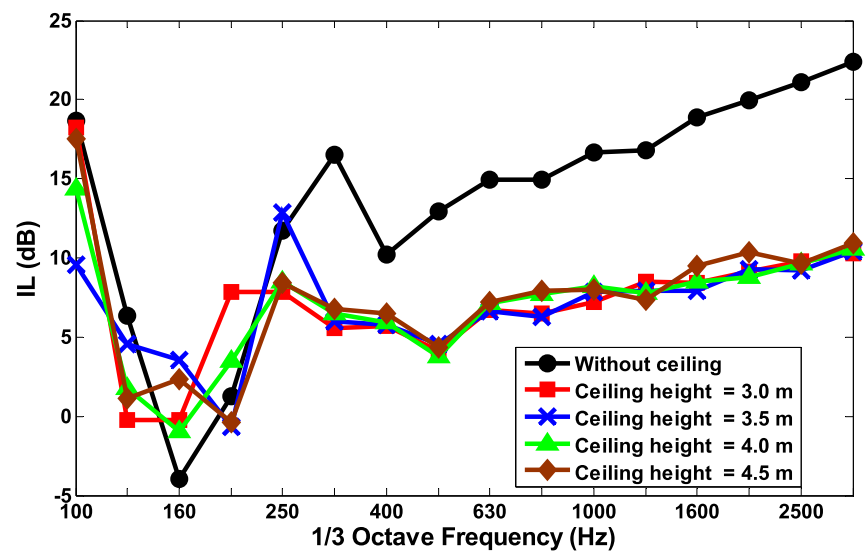

(b)

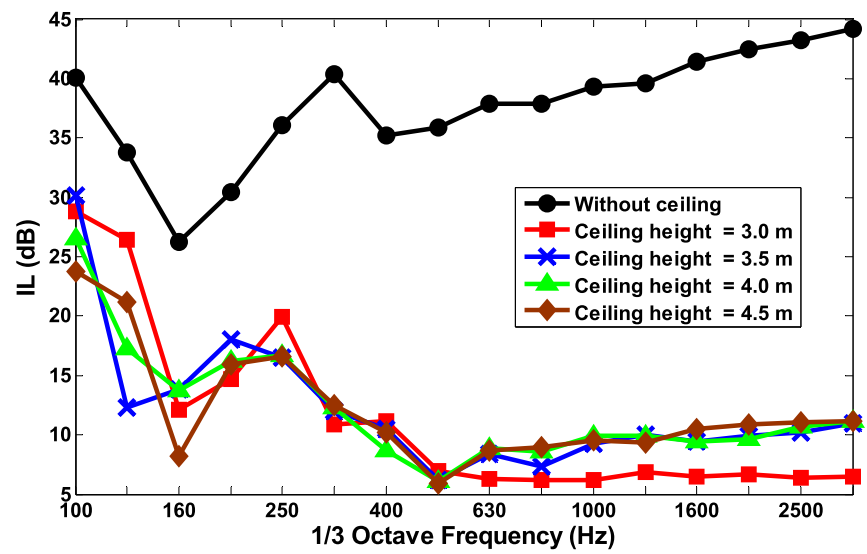

(c)

Fig. 8. Performance of the passive and active noise barrier at different ceiling heights: (a) sound pressure level without barrier; (b) insertion loss of the passive barrier; (c) insertion loss of the active barrier. coefficient makes little improvement on extra insertion loss provided by the active system in high frequency, as shown in Fig. 7(b) and (c).

Variations of the SPLs and ILs with different ceiling heights are not significant for the frequency above $250 \mathrm{~Hz}$, but have some changes below $250 \mathrm{~Hz}$, as shown in Fig. 8. Similarly, no matter what the ceiling height is, with the existence of the ceiling, the deterioration of passive barrier performance is larger than $10 \mathrm{~dB}$, and the performance of the active barrier deteriorates more. The ILs seems not sensitive to the change of the ceiling height, and the variation does not have a clear trend. In the simulations, the flow resistance of the ceiling is $100 \mathrm{cgs}$, and other parameters have the same values as that in Section 2.

\subsection{The effect of the sensing strategy}

The SPL of the indoor passive and active noise barrier in an enclosure with 3 different error sensor strategies are shown in Fig. 9, where "Error sensor near barrier" means error sensor is placed in front of the secondary source with a distance of $0.2 \mathrm{~m}$, "Error sensor at observation location" means error sensor is placed at the centre of the observation area, and "Ideal sensing" means minimizing the squared sound pressure of the entire observation area. All parameters in this subsection have the same values as that in Section 2.

Fig. 9 shows that different error sensor strategy has different performance. Changing error sensor strategies do not improve the active performance significantly at frequency larger than $500 \mathrm{~Hz}$. At frequency below $500 \mathrm{~Hz}$, the difference between different error sensing strategies is large, and the system can achieve 5-20 dB insertion loss with the ideal sensing. However, this strategy is difficult to implement in reality. When the only error sensor is located at the centre of the observation area instead of using the ideal sensing, the effective bandwidth shrank to $250 \mathrm{~Hz}$, and its performance at high frequency performance deteriorate significantly. Putting the only error sensor in front of the secondary source with a distance of $0.2 \mathrm{~m}$ can have similar insertion loss as that when the only error sensor is located at the centre of the observation area.

Figs. 10 and 11 show the $1 / 3$ octave band sound field distribution with the error sensor located at the observation area centre for $250 \mathrm{~Hz}$ and $2000 \mathrm{~Hz}$ respectively. As shown in Figs. 10(c) and 11(c), putting the error sensor at the observation area centre can form a

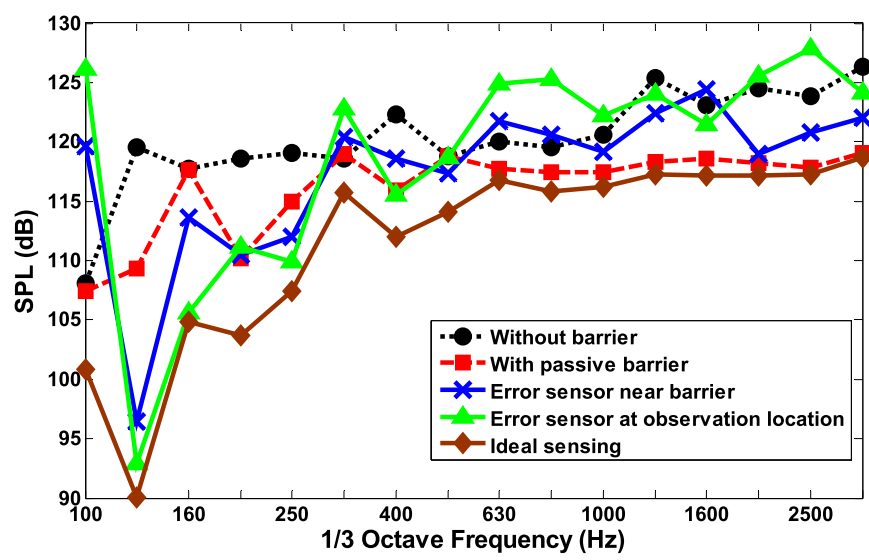

Fig. 9. Performance of the active noise barrier at different error sensor locations, where "Error sensor near barrier" means error sensor is placed in front of the secondary source with a distance of $0.2 \mathrm{~m}$; "Error sensor at observation location" means error sensor is placed at the centre of the observation area; "Ideal sensing" means minimize the squared sound pressure of the entire observation area. 
"quiet zone" around the error point, the higher the frequency is, the smaller the zone will be. It is difficult to obtain good performance in the entire observation area in the high frequency range if there is only one error sensor. Minimizing the squared sound pressure of the entire observation area can have larger quiet zone at higher frequency.
For the sensing strategies discussed above, the "Ideal sensing" strategy and the "Error sensor at observation location" strategy are not feasible in practical situations because the person's head will be around the observation location. The "error sensor near barrier" might be a practical solution. The performance of using the error sensor located in front of the secondary source at a distance of

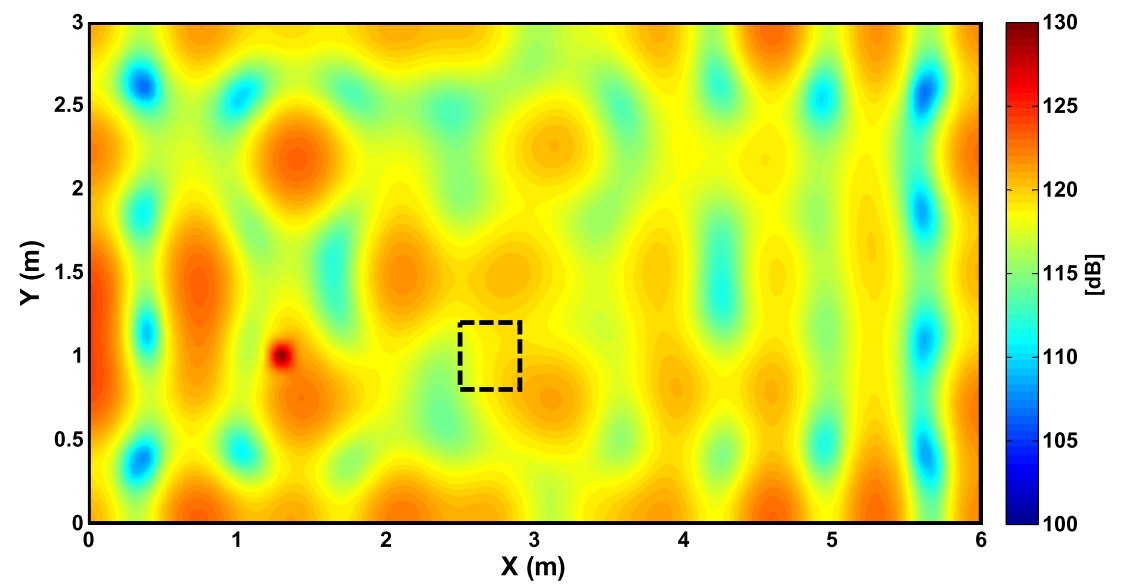

(a)

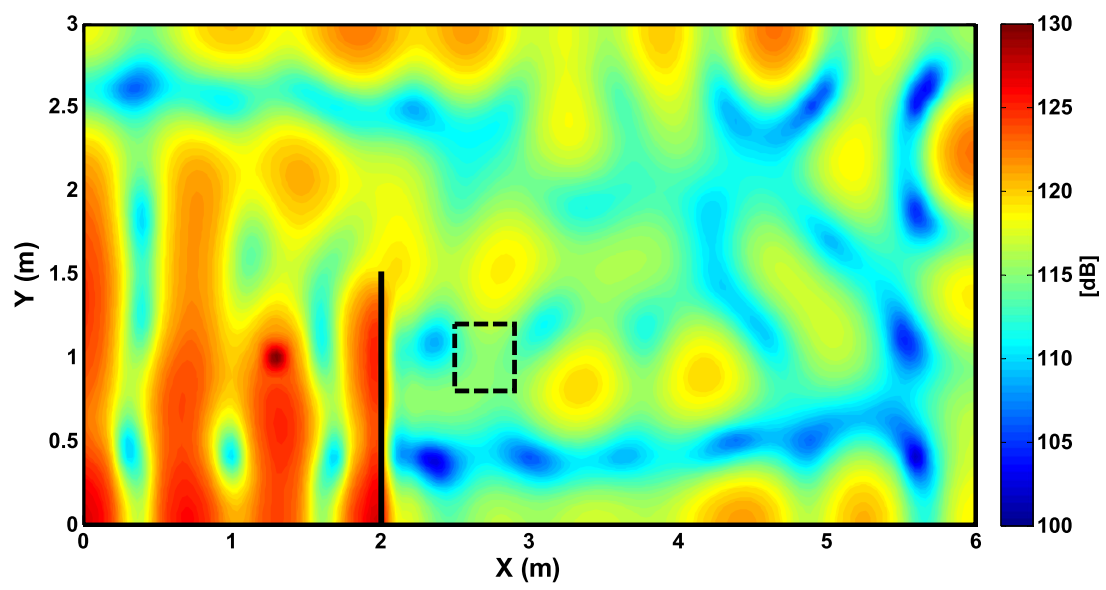

(b)

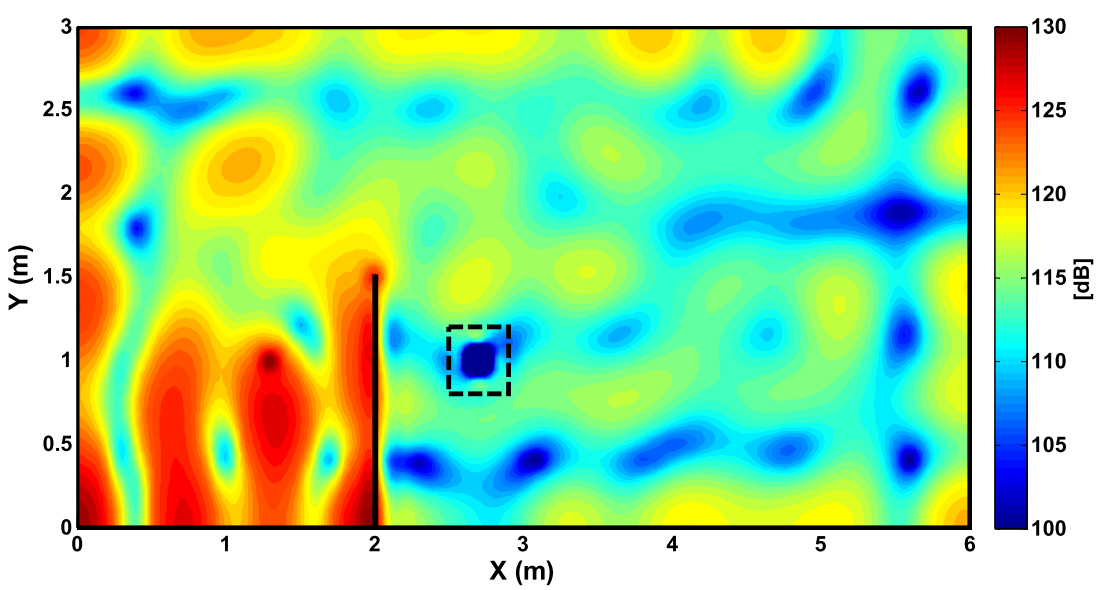

(c)

Fig. 10. Sound field in the room at $250 \mathrm{~Hz} 1 / 3$ octave band (a) primary sound field (b) with passive barrier only (c) with active barrier, the observation area is surrounded by dotted line. 
$0.05 \mathrm{~m}, 0.1 \mathrm{~m}$ and $0.15 \mathrm{~m}$ away is shown in Fig. 12, where it is observed that the performance does not change significantly. That indicates that slight changes of the error sensor location near barrier cannot change the active barrier performance significantly. Being compared with the "Ideal sensing", the active barrier performance deteriorates significantly when locating the error sensor near barrier. The extra insertion loss only increases a small amount in the low frequency range.

Regarding the error sensor near barrier, there are also other strategies can be applied such as the virtual sensing strategy. To overcome the inconvenience of placing the error sensor at the desired location, a number of virtual sensing algorithms have been

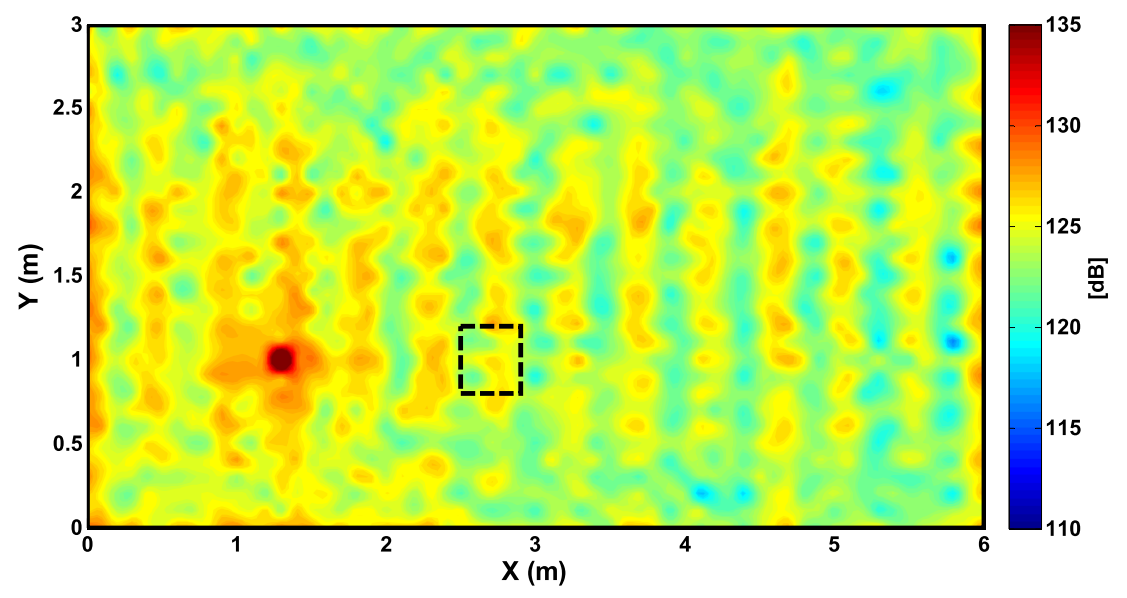

(a)

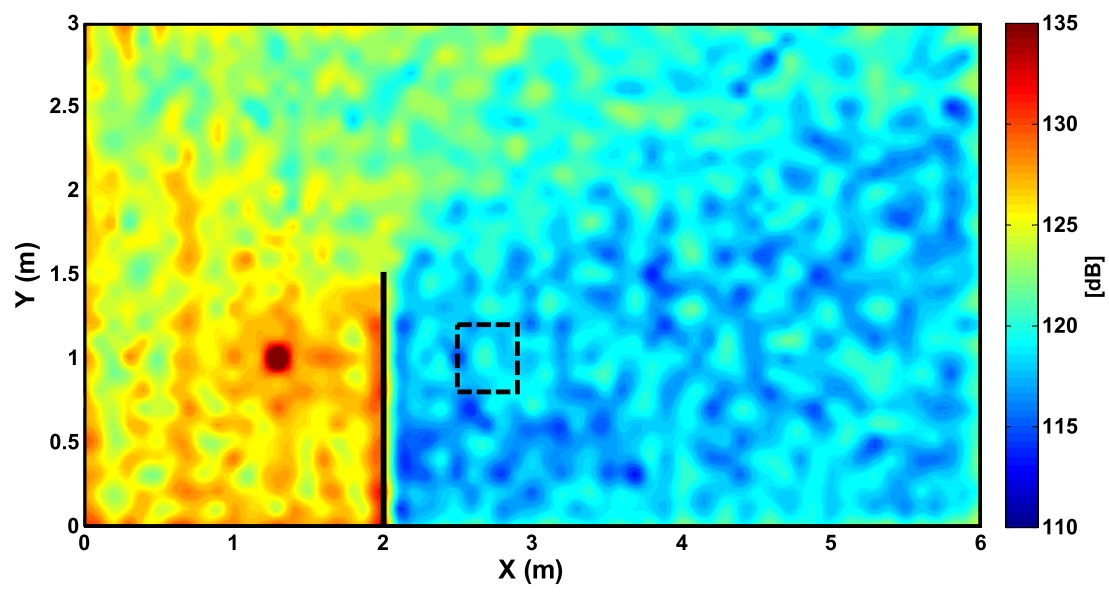

(b)

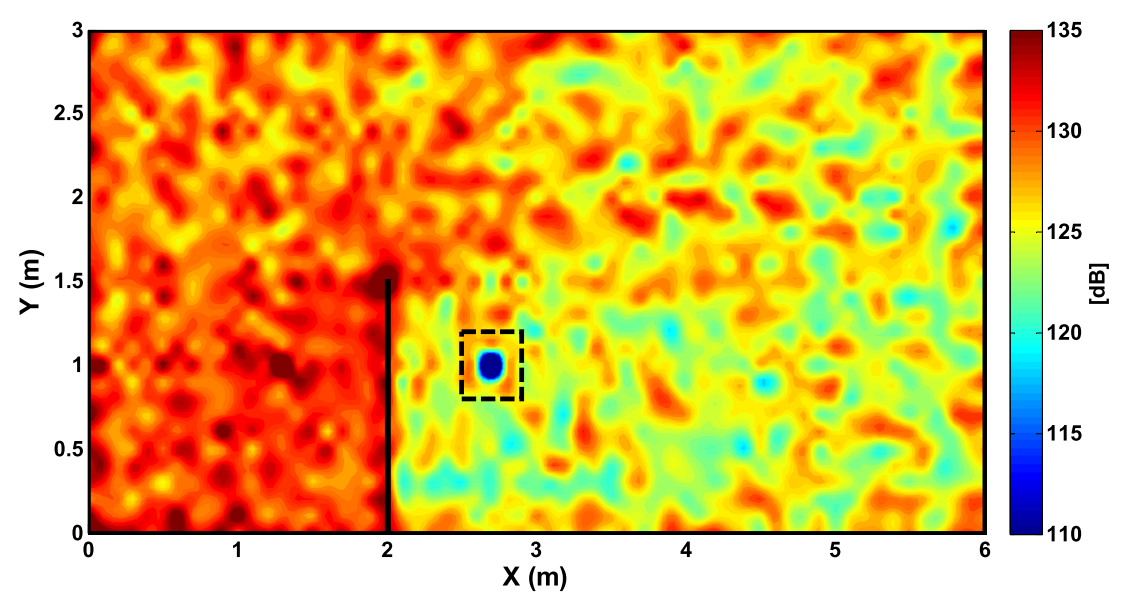

(c)

Fig. 11. Sound field in the room at $2000 \mathrm{~Hz} 1 / 3$ octave band (a) primary sound field (b) with passive barrier only (c) with active barrier, the observation area is surrounded by dotted line. 


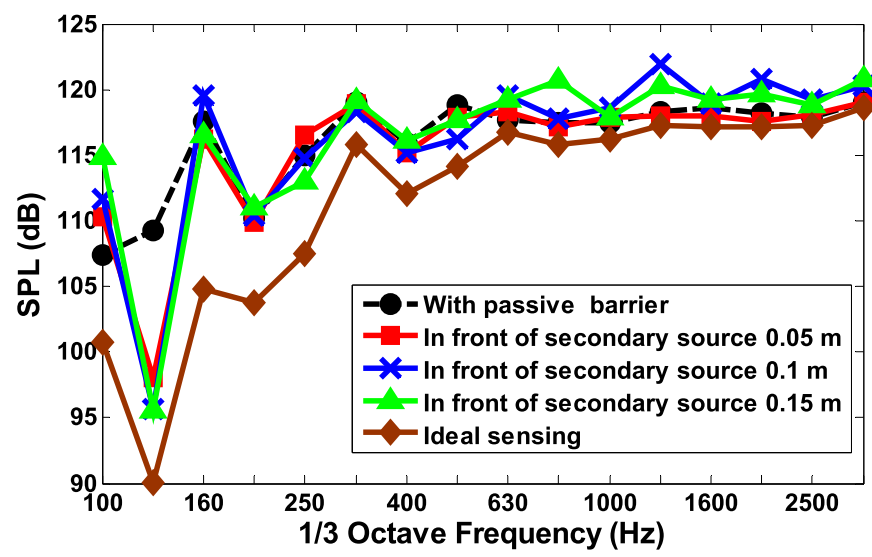

Fig. 12. Performance of the active noise barrier when error sensor located near barrier.

developed for active noise control [25], which use the physical error signal, the control signal and knowledge of the system to estimate the error signal at a location that is remote from the physical error sensor. Instead of minimizing the physical error signal, the estimated error signal is minimized with the active noise control system to generate a zone of quiet at the virtual location.

A number of virtual sensing algorithms have been developed and experimentally investigated to estimate the pressure at a fixed virtual location [26]. However, it is likely that the desired location is not fixed in practical situations, so some moving virtual sensing algorithms have been proposed to generate a virtual microphone capable of tracking a moving virtual location [27]. When combined with an active noise control algorithm, these moving virtual sensing algorithms were shown to achieve greater attenuation at the moving virtual location than control at a fixed physical or virtual sensor [27].

Another strategy can be used is minimizing the sound intensity at the error sensor locations. Sound intensity has been chosen as the cost function to obtain the extra sound insertion loss in the dark area of a hybrid active noise barrier system and off-line experiments for a three-channel control system show the active sound intensity control is able to provide better far-field noise reduction than the squared sound pressure control [28].

The virtual sensing strategy and minimizing the sound intensity have the potential to improve the performance; however, they are not discussed in the manuscript because there are many aspects for implementing the strategy which are beyond the scope of the current manuscript.

Although the research of this manuscript is based on a $2 \mathrm{D}$ model, these conclusions are useful in some 3D cases, for example, in a large open-plan office or a room with large side-wall absorption. Unfortunately, the conclusions derived from 2-D simulation cannot be extended simply to the 3D cases because many other factors should be considered in 3D cases. These include the side wall reflections, the number of secondary source and error sensor, and the spacing between two adjacent secondary sources. A 3D model for indoor active noise barriers needs to be developed for next stage research.

\section{Conclusion}

The feasibility of using active control systems to improve the performance of indoor barriers is investigated in this paper based on 2D numerical simulations. The performance of the indoor passive and active noise barrier is investigated first in a typical room by comparing the sound pressure level in the observation area under different circumstances. Then, the influence of the reflecting boundaries is examined by comparing the SPL and insertion loss with different reflection conditions. Further simulations are carried out in a flat room by changing the normal incident absorption coefficient of the ceiling and the height of the room to determine the effect of the ceiling reflection. Finally, three different error sensor strategies are proposed and discussed to understand the effect of the sensing strategies.

The simulation results show that about $10 \mathrm{~dB}$ extra insertion loss can be obtained below $500 \mathrm{~Hz}$ by using the active control system with the ideal error sensing strategy. Nevertheless the impact of the indoor active noise barrier on the area of the source side is small, and the secondary sound generated by the active control system should not be noticed by the persons there. The noise reduction performance of the indoor noise barrier is smaller than that of the same barrier in semi-free field due to the reflections from the ceiling. Also because of the ceiling reflection, the performance of the active control system is not sensitive to the normal incident absorption coefficient of the ceiling and the height of the room. For practical applications, the error sensor might be put near the secondary source on the barrier, which causes the effective bandwidth of active control to be reduced to a lower frequency of less than $250 \mathrm{~Hz}$. The main conclusion of the paper is that active control systems can be used to improve the performance of indoor barriers in low frequency range; however, the ceiling reflection and error sensor strategies need to be considered carefully during design to obtain the expected performance.

Despite the valuable findings, the conclusions obtained in paper does not take into account the reflections and effects from sidewalls, so a 3D model for indoor active noise barriers needs to be developed for next stage research. Further work also includes exploring other sensing strategies for improving the indoor barrier performance, such as the virtual sensing strategy and the sound intensity minimization.

\section{Acknowledgements}

Project 11474163 supported by National Natural Science Foundation of China.

\section{References}

[1] N. Han, C.M. Mak, A further study of a mathematical model for a screen in open-plan offices, Appl. Acoust. 69 (11) (2008) 1114-1119.

[2] A. Omoto, K. Fujiwara, A study of an actively controlled noise barrier, J. Acoust. Soc. Am. 94 (1993) 2173-2180.

[3] A. Omoto, K. Takashima, K. Fujiwara, Active suppression of sound diffracted by a barrier: an outdoor experiment, J. Acoust. Soc. Am. 102 (1997) 1671-1679.

[4] J. Guo, J. Pan, Increasing the insertion loss of noise barriers using an activecontrol system, J. Acoust. Soc. Am. 104 (1998) 3408-3416.

[5] J. Shao, J.Z. Sha, Z.L. Zhang, The method of the minimum sum of squared acoustic pressures in an actively controlled noise barrier, J. Sound. Vib. 204 (1997) 381-385.

[6] J.B. Moreland, J.R. Musa, The performance of acoustic barriers, Noise Control Eng. 1 (2) (1973) 98-101.

[7] S.K. Lau, S.K. Tang, Performance of a noise barrier within an enclosed space, Appl. Acoust. 70 (1) (2009) 50-57.

[8] S. Kopuz, N. Lalor, Analysis of interior acoustic fields using the finite element method and the boundary element method, Appl. Acoust. 45 (3) (1995) 193-210.

[9] A. Le Bot, A. Bocquillet, Comparison of an integral equation on energy and the ray-tracing technique in room acoustics, J. Acoust. Soc. Am. 108 (4) (2000) $1732-1740$

[10] E. Reboul, A. Le Bot, J. Perret-Liaudet, Radiative transfer equation for multiple diffraction, J. Acoust. Soc. Am. 118 (3) (2005) 1326-1334.

[11] P. Chevret, Advantages of the incoherent uniform theory of diffraction for acoustic calculations in open-plan offices, J. Acoust. Soc. Am. 137 (1) (2015) 94-104.

[12] C. Wang, J.S. Bradley, A mathematical model for a single screen barrier in open-plan offices, Appl. Acoust. 63 (8) (2002) 849-866.

[13] C. Wang, J.S. Bradley, Prediction of the speech intelligibility index behind a single screen in an open-plan office, Appl. Acoust. 63 (8) (2002) 867-883. 
[14] P. Chevret, J. Chatillon, Implementation of diffraction in a ray-tracing model for the prediction of noise in open-plan offices, J. Acoust. Soc. Am. 132 (5) (2012) 3125-3137.

[15] J. Yang, W.S. Gan, On the actively controlled noise barrier, J. Sound. Vib. 240 (2001) 592-597.

[16] A.P. Berkhoff, Control strategies for active noise barriers using near-field error sensing, J. Acoust. Soc. Am. 118 (2005) 1469-1479.

[17] W.R. Short, Global low frequency active noise attenuation, in: INTER-NOISE 80 vol. 2, 1980, pp. 695-698.

[18] P. Joseph, S.J. Elliott, P.A. Nelson, Near field zones of quiet, J. Sound. Vib. 172 (5) (1994) 605-627.

[19] P.A. Nelson, S.J. Elliott, Active Control of Sound, Academic Press, 1991.

[20] B. Rafaely, Zones of quiet in a broadband diffuse sound field, J. Acoust. Soc. Am. 110 (1) (2001) 296-302.

[21] M. Pawelczyk, Adaptive noise control algorithms for active headrest system, Control Eng. Pract. 12 (9) (2004) 1101-1112.
[22] Multiphysics C, COMSOL Multiphysics User Guide (Version 4.4), COMSOL, 2013.

[23] W.J. Hadden Jr., A.D. Pierce, Sound diffraction around screens and wedges for arbitrary point source locations, J. Acoust. Soc. Am. 69 (5) (1981) 1266-1276.

[24] K. Attenborough, Ground parameter information for propagation modeling, J. Acoust. Soc. Am. 92 (1992) 418-427.

[25] D. Moreau, B. Cazzolato, A. Zander, C. Petersen, A review of virtual sensing algorithms for active noise control, Algorithms 1 (2) (2008) 69-99.

[26] D. Moreau, J. Ghan, B. Cazzolato, A. Zander, Active noise control with a virtual acoustic sensor in a pure-tone diffuse sound field, in: Proceedings of the 14th International Congress on Sound and Vibration, 2007.

[27] C. Petersen, Optimal Spatially Fixed and Moving Virtual Sensing Algorithms for Local Active Noise Control, PhD thesis, School of Mechanical Engineering, The University of Adelaide, 2007.

[28] N. Han, X. Qiu, A study of sound intensity control for active noise barriers, Appl. Acoust. 68 (2007) 1297-1306. 